\title{
Elite running performances: the result of genetics, epigenetics and nurture
}

\section{Opinion}

Elite runners both sprinters and endurance runners seem to be restricted mostly to African race. There were researches on genetics and running performances of African runners with some favoring the concept of genetic predisposition of African runners whereas some others finding no such genetic link. This is a widely contested area of sports science and needs extensive research and intellectual reasoning. Though the genetic code of the human being was identified, the field of genetics is still has a long way to go in establishing the precision biodynamic of genetic interactions. The claim that certain level of consensus among scientists that the African runners are not predisposed with genetic endowment for running needs to be noticed and to be verified further. The fact is there is no single white or other race sprinter in men's 100 meters finals of Olympics since 1984. How can this happen if there is no genetic disposition for African runners, especially West African race runners towards sprinting. If only epigenetic factors like the training modules, diet, environment etc are playing role in this, the same may also can be adapted by other race runners. The Kalenjin tribe phenomenal success in distance running needs to be examined in terms of both genetics and epigenetic to try to find some reasonable answer for this long discussed but still inconclusive topic. The same is true with Ethiopian runners too. There may be somatotype advantages for these runners over European and other race runners as some are reasoning, like lighter calves and thinner and long legs, which again need to be ascribed to genetic adaptation to chronic environmental, activity stimuli which could have provided such advantage to move swiftly and to run for longer durations. But, another fact is that such somatotype advantages are there for many other African tribe and European tribe runners. ${ }^{1}$

The concept of human myokine has revolutionarised the thought process about the genetics of physical activity and exercise adaptations both in disease and sports performance areas. It has been found that the skeletal muscle secretes several hundreds of hormone like protein substances which cross talk with several organs of the body and are very vital for maintenance of the biochemical environment of the organism. Many of these signaling myokine act as activators and transcriptors of genes for proper expression. Peroxisome-proliferator activated receptor $\gamma$ co activator $1 \alpha$ (PGC- $1 \alpha)$ myokine acts on the PGC- $1 \alpha$ gene regulates the mitochondrial genes thereby bringing changes in the endurance abilities of individuals. Studies proved that the chronic endurance training improves this transcript levels in PGC$1 \alpha$ gene thereby enhancing the mitochondrial functionality. ${ }^{2}$ There may be some subtle differences in terms of quality and quantity of this myokine that differentiates the genetic orientation of runners towards endurance running. In the same way myokine IL-15 (Interleukin 15) and mitochondrial genetics need to be interlinked to understand the subtle inter dependency in making individuals more favorable to endurance running. Muscle specific ablation of this IL-15 in some studies could favor that IL-15 specificity could bring differences in oxidative capacities of individuals. Studies also supported about the remodeling of muscle tissue due to chronic exercise or physical activity related IL-15 dynamics. Gene is not something static and
Volume I Issue I - 2017

\author{
Rajasekhar Kali Venkata \\ Director of Physical Education and sports, University of \\ Hyderabad, India
}

Correspondence: Rajasekhar Kali Venkata, Director of Physical Education and sports, University of Hyderabad, India, Email jatinsriraj@yahoo.co.in

Received: May 13, 2017 | Published: May 16, 2017

genetic changes could occur due to several stimuli and physical activity stimulus is one major factor that has influenced the human genetics over millions of years. Another myokine BDNF (Brain Derived Neurotrophic Factor) generally expressed in brain, which regulates the neuro plasticity also is produced in muscle during muscular contraction. Muscle origin BDNF plays vital role in myogenesis and muscle cell regeneration, which plays vital role in post run recovery. Another myokine Irisin, which is cleaved off from a muscle protein called FNDC5 has effective role in neurogenesis and also cause for higher expression of PGC- $1 \alpha$, mitochondrial transcription factor and glucose transporter 4 and thereby enhancing the aerobic metabolic status of individual. Irisin secretion in skeletal muscle during the physical activity depends on the type, intensity, duration etc and hence expression of mitochondrial genes may accordingly change. Epigenetics need to be the result of genetic predisposition and stimuli like physical exercise, exposure to high altitude; environment, diet etc are associated as epigenetic factors. But, nurturing of athletes with proper training protocols, nutrition programs, rest, recovery etc is equally important to make individuals who are genetically endowed as elite performers. Epigenetics may answer this concept of nurturing for elite performances. It is also true that chronic exposure to physical training would lead for adaptations leading to enhanced performances. ${ }^{3}$

A simple analogy that genetic risk to some diseases and difference of black and white skin between African and European races a genetic variation and this simple understanding could certainly pave way for a strong belief that there may be genetic predisposition among different human races with different physical capacities basing on the stimuli they were exposed for generations. A broader spectrum of subtle inter dependent issues of genetics and epigenetics would provide a logical understanding that certain human races like Kalenjin tribe may be predisposed with genetic advantage for distance running.

\section{Acknowledgements}

None.

\section{Conflict of interest}

Author declares there is no conflict of interest in publishing the article. 


\section{References}

1. Vancini RL, Pesquero JB, Fachina RJ, et al. Genetic aspects of athletic performance: the African runners phenomenon. Open Access $J$ Sports Med. 2014;5:123-127.
2. Wilber RL, Pitsiladis YP. Kenyan and Ethiopian Distance runners: what makes them so good? Int J Sports Physiol Perform. 2012;7(2):92-102.

3. Deason M, Scott R, Irwin L, et al. Importance of mitochondrial haplotypes and maternal lineage in sprint performance among individuals of West African ancestry. Scand J Med Sci Sports. 2012;22(2):217-23. 